\title{
LOS ESTUDIOS DE POSGRADO EN CIENCIAS SOCIALES EN CHILE: ALGUNAS CONSIDERACIONES CRÍTICAS
}

\author{
Jorge Larraín*
}

* Director del Departamento de Ciencias Sociales de la Universidad Alberto Hurtado. 


\section{RESUMEN}

El artículo analiza críticamente algunos aspectos de la enseñanza de posgrado en ciencias sociales en Chile, especialmente su larga duración y su lectividad excesiva. Para este análisis se toma un punto de vista comparativo, contrastando el sistema británico con el chileno. Mientras en Gran Bretaña el taught master es un paso intermedio entre el primer grado y el doctorado, en Chile las maestrías tienden a repetir la estrategia de las carreras. Se argumenta que las maestrías deberían hacer más énfasis en los seminarios como método de aprendizaje para propender a la lectura personal, la presentación de ponencias y ensayos y, en definitiva, para fomentar el desarrollo de las cualidades críticas y la autonomía de pensamiento de los alumnos. Todas estas competencias son indispensables, sea para poder desarrollar investigaciones más tarde, o para integrarse a la vida profesional con mejores herramientas.

\section{ABSTRACT}

This article contains a critical analysis of some aspects of the postgraduate teaching of Social Sciences in Chile, especially their excessive length and extreme reliance on courses. The analysis is based on a comparative approach contrasting the British system and the Chilean one. While in Britain the taught master is an intermediate step between the first degree and the doctorate, master's degrees in Chile tend to repeat the strategy of first degrees. It is argued that masters should emphasize seminars as a learning method in order to favor individual reading, presentation of papers and essays and, ultimately, to develop critical qualities and independence of mind on the part of students. All these skills are indispensable, be it for developing research later on, be it for pursuing a professional life with better tools. 


\section{LOS ESTUDIOS DE POSGRADO EN CIENCIAS SOCIALES EN CHILE: ALGUNAS CONSIDERACIONES CRÍTICAS}

Quiero contribuir con algunas reflexiones generales sobre los posgrados en ciencias sociales en Chile, especialmente las maestrías. Mis pensamientos no son fruto de una investigación rigurosa, sino más bien de mi propia experiencia de algunos años observando y participando en el sistema universitario chileno y de muchos más como miembro del sistema universitario británico. Es posible que algunas opiniones sean muy debatibles, sesgadas o provocativas, quizás fruto de una experiencia limitada a solo algunas universidades chilenas y de mi anglofilia académica, que declaro abiertamente, pero de todos modos las entrego con el objeto de fomentar la reflexión y el diálogo sobre este importante aspecto de la vida universitaria. No pretendo excluirme ni excluir a mi propia universidad de este escrutinio crítico; analizo lo que es un sistema establecido en Chile, del cual nadie puede fácilmente sustraerse. Debido al carácter personal de mi contribución no se encontrarán notas ni referencias a estudios o investigaciones sobre el tema. Más bien se trata de impresiones personales nutridas por la comparación entre Chile y Gran Bretaña.

Antes de empezar conviene aclararle un poco más al lector desde dónde hablo para que mis observaciones hagan sentido. Soy un académico en ciencias sociales y la mayor parte de mi carrera ha transcurrido en la Universidad de Birmingham, Inglaterra (en sociología y estudios culturales) desde 1977 hasta 1995, fecha en la que me reintegré a la universidad chilena, aunque he seguido enseñando tres meses del año en Birmingham hasta el año pasado. Me fui de Chile en 1973 cuando el sistema universitario chileno contaba con pocas universidades y todas de carácter estatal. Volví cuando estaban en pleno auge las universidades privadas, pero el sistema estatal todavía conservaba una presencia decisiva. 
Cuando me fui casi no había posgrados de ciencias sociales en Chile. Todo tenía que hacerse en el extranjero. Yo mismo empecé haciendo mi máster en la Universidad de Sussex y seguí después al doctorado. Cuando volví, la situación había cambiado radicalmente: muchas universidades tenían el grado de máster en varias disciplinas y algunas incluso programas de doctorado. Esto era algo que tenía que suceder y es bueno que haya acontecido. Junto con la maduración del sistema universitario chileno era crucial que se desarrollaran los posgrados para ponernos al día en un área fundamental de toda universidad y para evitar que aquellos que quisieran cursarlos tuvieran que ir al extranjero, lo que es caro y difícil de lograr.

Sin embargo, como en muchas cosas en las que intentamos ponernos al día, las hacemos tarde y sobredimensionadas para nuestras necesidades. Ya en 1973, fecha en la que me incorporé, como estudiante de doctorado, al sistema universitario británico, Chile tenía un atraso considerable en las tendencias de pregrado existentes en esa época: mientras nosotros seguíamos con carreras de cinco o seis años, en el mundo anglosajón los primeros grados (o carreras como las llamamos nosotros) habían sido reducidos a tres años. Treinta años después, mientras la Comunidad Europea está uniformando esta modalidad para todos sus miembros, Chile sigue en lo mismo, con carreras excesivamente largas y sobredimensionadas para las necesidades del país.

No es sorprendente entonces que al incorporar los posgrados el sistema universitario chileno lo haya hecho siguiendo esta misma tendencia: excesivamente largos, excesivamente lectivos y todo esto sancionado por ley, de modo que ninguna universidad puede cambiar el sistema. Mientras en Gran Bretaña es posible hacer un máster en un año, aquí en Chile tenemos que hacerlo en dos años o más. Mientras en otros lados un máster exige la participación en tres o cuatro seminarios durante dos trimestres o seis meses, aquí en Chile tenemos dieciocho meses de intensa lectividad, más que quintuplicando las exigencias de cursos.

El excesivo énfasis en cursos, que hace a la maestría parecerse a una carrera breve, va acompañado de una curiosa actitud dual con respecto a la tesis de grado. Por una parte, esta no pareciera ser parte 
esencial de los estudios. Hasta hace muy poco, en muchas maestrías no había plazo de entrega de la tesis, y el resultado era que muy poca gente la hacía. Muchos alumnos se contentaban con pasar la valla de los cursos. Pero cuando se llegaba a la tesis, ya no había ánimo, ni tiempo, ni paciencia para hacerla. Algunas maestrías de universidades prestigiosas de Chile han tenido en esto un récord lamentable: el porcentaje de titulados ha sido ínfimo. En otros países, en cambio, la tesis de grado está perfectamente integrada y debe entregarse dentro del plazo del año que dura el máster, so pena de perder el grado. Por otra parte, existe también una expectativa sobredimensionada sobre las tesis. Se espera que tengan muchas páginas y se rodean de solemnidades propias del doctorado como es, por ejemplo, la defensa oral de la tesis. De modo que las maestrías en Chile están tensionadas entre una parte lectiva que se concibe como un pequeño pregrado y una parte de trabajo personal de tesis que se concibe como un pequeño doctorado. No hay continuidad entre las dos. De la parte lectiva está ausente el énfasis en la investigación, el trabajo personal y las ponencias en seminarios; en la tesis, en cambio, se exigen las competencias en las que no se preparó a los alumnos en la parte lectiva y se pide más de lo que sería esperable en un posgrado inicial. Por eso la tesis se transforma para muchos en una valla insalvable.

En Inglaterra al nivel de posgrado existe la distinción entre taught degrees y research degrees. Un taught master dura normalmente un año, mientras que, dentro de los research degrees, un master by research (M. Phil) dura dos años y un doctorado (Ph. D), tres años. Es decir, hay también maestrías con énfasis en la enseñanza por cursos. Pero ellos duran normalmente un año y su lectividad es equivalente a tres o cuatro seminarios en seis meses. Nuestras maestrías, en cambio, aunque duran dos años, no equivalen a un research degree, sino que son taught masters sobredimensionados con cinco veces más cursos y muy poco énfasis en la investigación y el trabajo personal. En mi experiencia personal uno de los sentimientos más comunes en estudiantes de maestrías en Chile es la frustración de haber abordado someramente muchas cosas, pero no haber tenido tiempo para leer más y entrar más a fondo en algunos temas de su interés.

El resultado de esto es que nuestras maestrías no constituyen ese paso intermedio entre la enseñanza por cursos de una carrera y la 
investigación avanzada de un doctorado. Tienden a repetir el procedimiento de la carrera y no preparan a los estudiantes sea para la investigación, sea para una vida profesional con mejores herramientas y competencias. Un máster debiera servir a los alumnos como un paso previo a la investigación o a la vida profesional, porque fomenta el trabajo personal, la lectura, la discusión crítica y la toma de posiciones, todos elementos claves para ir formando la independencia de juicio que todo investigador o profesional debe tener. Los cursos de nuestras maestrías tienen raras veces las características de seminarios donde los alumnos deben presentar trabajos maduros, fruto de sus lecturas personales y donde la discusión, propia de un seminario, es el centro de la enseñanza. Mi experiencia es que los cursos de maestría siguen siendo verticales, todo se espera del profesor, hay poca discusión o debate, y aún menos trabajo personal basado en lecturas profundas.

La forma de organización de las maestrías con respecto a la participación de los alumnos connota una falta de confianza en la madurez de los candidatos y en su capacidad para asumir la responsabilidad de leer, pensar y escribir ensayos y ponencias sin tener que estar largas horas oyendo la exposición efectuada por el profesor. Nuestras maestrías parecen no estar diseñadas para que los estudiantes aprendan a pensar por sí mismos. Son todavía muy escolares y rígidas y no se diferencian mucho de las carreras de pregrado. ¿Cuántos de nuestros cursos de posgrado tienen realmente el carácter de un seminario en el que los propios alumnos participan activamente con presentaciones y discusiones en profundidad? Privilegiamos el número de materias cubiertas por el máster en desmedro de la profundidad con la que deberíamos tratar solo algunas pocas. La cantidad y la extensión están bien en el pregrado; en el posgrado hay que hacerse cargo de menos temas pero más a fondo.

Existe una tendencia a creer que la seriedad de un programa de posgrado está dada por su duración, su número de cursos y los títulos o "nombres" de sus profesores. Mientras más largo, con más cursos y profesores más conocidos o con más títulos, tanto más serio y prestigioso. Pero la calidad y la seriedad no está en relación directa con la cantidad en ninguno de estos aspectos. Sin duda es bueno tener profesores de prestigio y con muchos títulos, pero siempre que 
realmente pertenezcan al departamento que imparte el máster o doctorado y que estén disponibles para atender a los alumnos más allá de la hora de clase. Una de las cosas que más sorprende es que muchos de los académicos de programas de posgrado ni siquiera pertenecen al departamento que los imparte sino que han sido reclutados para poner sus nombres y títulos en los folletos publicitarios y parecer así de alto nivel. En verdad son profesores por hora que hacen una clase y se van. En esto tampoco hay muchas diferencias con los pregrados. Pero si bien en el nivel de pregrado esto es menos dañino porque allí se esperan clases más puntuales, más verticales y de menor interacción, en el nivel de posgrado es más grave. Los alumnos tienen poca oportunidad de encontrar al profesor para conversar, discutir, presentar borradores, $\mathrm{u}$ otras actividades que contribuyen a formarlos.

Se podría pensar que uno de los resultados negativos de la introducción de universidades privadas es su estilo de gestión que no privilegia los contratos por jornadas completas o medias y al contrario, mantienen un gran cuerpo de profesores hora. En parte esto es cierto. Pero esta tendencia también se ha ido imponiendo en las universidades estatales a pesar de que tienen un número mucho mayor de profesores de planta; y no podría ser de otro modo, dado el enorme número de cursos a impartir. No hay departamento que pueda tener expertos en tantos cursos como los que supone impartir un máster en los programas actuales.

¿Cómo mejorar las maestrías en Chile? De lo que he dicho se deducen algunos posibles cursos de acción. Primero, permitir que haya maestrías de un año, aunque sea posible hacerlo en dos en caso de alumnos part-time. Segundo, reducir drásticamente la lectividad de modo de tener no más de tres o cuatro seminarios en el año y que estos cubrieran solo los primeros seis o siete meses. Tercero, transformar los cursos en verdaderos seminarios donde los alumnos tengan una gran responsabilidad y participación. Evitar las clases magistrales y basar los seminarios en mucha lectura y discusión. Cuarto, darle mucha importancia a la tesis o disertación final pero integrada dentro del plazo del año de estudios (por ejemplo, el espacio para escribirla podría estar entre septiembre y noviembre) de modo que no pueda tomarse como algo adicional o extra. Si un máster empieza en marzo, la tesis debería estar lista en diciembre, salvo 
enfermedad $u$ otro problema que afecte al candidato. Pero, una vez más, hay que evitar exagerar las expectativas sobre la tesis: esta no debería exceder de veinte mil palabras ni debiera tener que defenderse públicamente. Es solo un primer ejercicio de posgrado y debe ser corto y preciso, pero tiene que demostrar las cualidades de una excelente organización, discusión y manejo de fuentes y datos junto con la independencia de juicio a que hacíamos alusión anteriormente. Quinto, tender a que las maestrías, así como los doctorados, sean impartidas por los académicos de planta de cada departamento y limitar la contratación de profesores hora. De este modo se facilitaría el acceso a los profesores y se podría tender a crear una verdadera comunidad de pensamiento y discusión entre docentes y alumnos. 